\title{
The fitness and health value of the Taijiquan Wujizhuang
}

\author{
Li Jing
}

\author{
Sect. Of P.E, Wuhan Univ. Of Tech., Wuhan 430070,China
}

Keywords: TaijioquanWujizhuang; Fitness value; Tiaoshen; Pranayama; Tiaoxin

\begin{abstract}
Taijiquan is gentle, slow and natural, which can make the body's material and energy be fully exchanged, thus improving the health value of human body. After the systematic combine of the fitness effect of the Taijiquan Wujizhuang, the function of 'Tiaoshen', 'pranayama' and 'Tiaoxin' was also found in the Taijiquan Wujizhuang. Taijiquan Wujizhuang has three functions of 'Tiaoshen ', 'Pranayama ' and 'Tiaoxin ' .Tiaoshen is refers to the internal function and external function to the body to adjust and improve, external function can improve the body quality, improve the ability of the muscle work, protection of the body, Internal organs can play the role of exercise organs the ability of various organs work; Pranayama refers to the adjustment of the body's breath. Through the adjustment of the breath, the blood circulation can be quickened, and the metabolism can be promoted to achieve the effect of circulation and circulation and prevent chronic diseases. The Tiaoxin is the adjustment of the psychological changes of the body, relaxed mood, the spirit is full, positive upward.It is the three-tone function of 'Tiaoshen ', 'pranayama ' and 'Tiaoxin ' to make the human body in a healthy and harmonious state.

Taijiquan, as one of outstanding national traditional sports events in China, is characterized by long history and clear heritage. It was successfully applied as the first batch of state-level nonmaterial cultural heritage on May 20, 2006. The State Council issued a series of documents related to improvement of national physical quality and promotion of national physical and mental health, etc. in succession since 1995 such as 'National Fitness Plan Outline', 'National Fitness Plan (20162020)', '<Healthy China 2030> Plan Outline'. Therefore, Taijiquan sports was developed rapidly, and the audience reached more than two hundred million. China is the birthplace of Taijiquan. In China, the elderly mostly practiced Taijiquan at first. Middle-aged people gradually joined the sports later, and young people, teenagers and children are involved in practicing Taijiquan in recent years because Taijiquan can exercise the body, strengthen physique, regulate inner loop and outer loop of human body, and achieve the effect of preventing and inhibiting and treating diseases. It has similar effect as traditional Chinese medicine, acupuncture, massage and other treatment means, which is beneficial for promoting healthy living. Taijiquan practitioners are distributed throughout all ages. It is obvious that Taijiquan sport has been integrated into our life. Foreign study showed that Taijiquan exercise can lower blood pressure, improve sleep quality, adjust life stress, etc. which is greatly beneficial for improving the physical and mental health of human body. Taijiquan is favored by more and more foreign people. Practitioner groups are larger and larger. People can prevent diseases, have the rehabilitation effect to some chronic diseases, and save medical expenditure. Taijiquan exercise begins from the standing, while Wujizhuang is the most basic standing exercise, Taijiquan Wujizhuang is one of the exercises for getting refined internally and externally. Participants can tone body and stay healthy by long-term exercise. It is characterized by simple action and convenient exercise, which belongs to static exercise. A large number of literature shows that Taijiquan Wujizhuang has the functions of Tiaoshen, Pranayama, Tiaoxin etc.
\end{abstract}

\section{Overview of Taijiquan standing exercise}

Standing exercise is one of the basic skills in traditional Wushu, and it is one of the methods for exercising physical stability and inner power. There is no clear definition about 'standing exercise' through reviewing books and literature. However, more valuable explanation is discovered. Standing exercise is defined as follows in 'Practical Complete Collection of Chinese Martial Arts': 
'standing exercise is an inner power exercise method which is static externally and dynamic internally, wherein static standing posture is kept for leading Qi with consciousnesses and moving the body with Qi' ${ }^{[1]}$. Standing exercise is explained as follows in 'Chinese Martial Arts Dictionary' that standing exercise is one of basic skills for exercising leg strength and step firmness, and it is mainly divided into house-riding style halt and flexed foot position' ${ }^{[2]}$. Standing step is explained as follows in 'Overview of Chinese Martial Arts' that participants exercise various step methods, thereby strengthening leg strength, they can stand firmly as if a pile planted in the earth, thereby it is named ${ }^{[3]}$. It is summarized as follows through book and literature review that Taijiquan Wujizhuang mainly refers to the long standing under the quiet attitude, and the body can be exercised for combining practice and recuperation through getting refined internally and externally under the state of solidifying body, reaching omphaloskepsis, breathing evenly, and keeping natural. It mainly aims at concentrating attention, enhancing body stability, and improving spirit and energy. Taijiquan Wujizhuang can temper idea quality and psychological quality, and open mind of people by 'mind concentration' and 'mental abridgment' in standing exercise. Taijiquan Wujizhuang benefits to the human body is divided into internal benefit and external benefit. Blood and Qi operation as well as working efficiency of muscle and livers can be improved internally through Qi and idea adjustment, thereby improving physiological function of all tissues and organs so as to reduce injury caused by internal organ impact and shock due to various factors. Limbs and muscles are exercised externally, muscle contraction and tension magnitude can be exercised through long-term standing exercise for improving the coordination power and flexibility of muscles, thereby playing the protective role to body ${ }^{[4]}$. 2. Fitness and health effect of Taijiquan Wujizhuang

It is mentioned in 'Huangdi Neijing' that the standing exercise is a kind of ancient health methods in China ${ }^{[5]}$. It can be obvious that people had the concept of health since ancient times, and the health effect to standing exercise was recognized. Practitioners make the body in a state with relatively static consciousness through mobilizing minds thereof namely Taijiquan 'thoughts' during practice of standing exercise. Such exercise plays the role of nourishing vigour, harmonizing Qi and blood, opening channels and collaterals, balancing Yin and Yang, culturing root, and solidifying Yuan of human body ${ }^{[6]}$. 'Mind' should reach omphaloskepsis during practice of standing exercise, namely 'destiny internal contraction' and 'spirit collection', and achieve 'no external release of spirit and no external flow of will', thereby obtaining the effect of 'nourishing spirit, Qi and energy', namely 'Tiaoshen, Pranayama and Tiaoxin' effect ${ }^{[7]}$.

\section{Taijiquan Wujizhuang's effect on 'Tiaoshen'}

Tiaoshen refers to regulation of body shape, including limbs and all organs in the body, and the body can be regulated mainly through interaction among muscle, bone, membrane and flesh ${ }^{[8]}$.

Practice of Taijiquan Wujizhuang can improve people's physical quality, improve body working ability, and improve their mental outlook. 'Fairness' is firstly required in Wujizhuang practice. 'The virtual spirit is stiffening' is emphasized in the first article of boxing theory. It is emphasized that the body should be in a straight line, which should keep a line from head to trunk, head and body should not be inclined, the body should be straight, the pressure of the spine can be reduced on the head, thereby improving the mental outlook and correct posture. Shoulder muscles are in a relaxed state when shoulder and elbow are dropped. Shoulder joint diseases can be reduced during long period of exercise. The following diseases can be rehabilitated and prevented, such as periarthritis and other diseases. 'Chest is backward, back is pulled, and abdomen is solidified.' is the most important. Currently, many diseases are caused by spinal flocculant arrangement disorder, dislocation, shortening of vertebral moment, etc. It is found through the master's thesis 'physiological biomechanical analysis of influence of Taijiquan standing action on spine biological bending' ${ }^{[9]}$ that the physiological curvature of spine can be reduced, spine can be extended, and back can be pulled during standing exercise, thereby improving the stress of the intervertebral disc, and such exercise has relief and rehabilitation role to cervical spondylosis, disc herniation, lumbocrural pain and other diseases. 'Waist loosening and hip folding' have the role of adjusting the body center of gravity, supporting upper body, solidifying lower body, two legs' strength should be 
even, the center of gravity is always in the middle rather than being deviated to one side. The stability of leg muscles and hips can be enhanced. It is necessary to cooperate with systematic coordinated development during practice, head, trunk and feet should be integrated for practice.

'Nothing is as good as silence, and no exercise is as good as standing' ${ }^{[10]}$. Taijiquan Wujizhuang can adjust body internal Qi and blood as well as work of liver. Wujizhuang standing has the following requirements: natural static state, loose shape and concentrated will, fair comfort, external silence and internal movement as well as omphaloskepsis. The seemingly motionless body leads Qi with idea through concept guidance, movement is controlled by Qi, and mutual movement among muscles and joints in the body is promoted. Many diseases are caused by the imbalance of Yin and Yang in the body, thereby leading to Qi and blood obstruction as well as disorder of main and collateral channels. Qi, blood, main and collateral channels, liver and other organs should be uniform in order to adjust the balance of Yin and Yang in the body. The body is in the static state during standing exercise, the nervous system is rested when the body is relaxed, the muscle tension is also reduced, thereby accelerating blood circulation, Qi can be lowered to the pubic region for repeated breathing by cooperating with deep breathing, which not only can promote blood circulation, but also can increase the body strength. The exercise has significant regulation function for promoting Qi, activating blood, clearing and activating the channels and collaterals, thereby preventing, adjusting and treating cardiovascular system diseases, etc.. Abdominal breathing method can regulate Qi, strengthen the working ability of internal organs, and improve the body quality because of the special breathing mode of Taiji Wujizhuang-abdominal breathing method. The diaphragmatic muscle is stressed, the range of motion is increased for massaging the internal organs during abdominal breathing. The working ability of internal organs can be improved through 'massage' of internal organs. The movement ability of internal organs is also practiced during longterm abdominal breathing, thereby nourishing the internal organs. It is concluded in TCM that the quality of human body internal heart, liver, spleen, lung and kidney can be reflected outside the body, such as mental state, skin color, etc. Healthy work of internal organs can provide people with brand-new mental outlook. The limbs can be strong and flexible, thereby laying a solid foundation for the health of the body.

\subsection{Taijiquan Wujizhuang's effect to 'Pranayama'}

Pranayama means to breath adjustment. 'Adjustment' has the meaning of harmonization, adjustment and regulation. There are three ancient meanings of the word 'Xi':(1) spirit (2) breath (3) standstill among breaths ${ }^{[11]}$.

Taijiquan Wujizhuang breathing mode is mainly based on abdominal breathing. Abdominal breathing is divided into natural breathing and inverse breathing. Natural breathing refers that the abdominal muscle is expanded outwards slowly during inhalation under the conditions of breathing. More oxygen can be absorbed as far as possible under the condition of own comfort, and the gas can be slowly exhaled; Reverse breathing refers that abdominal muscle is contracted inwards slowly during inhalation under the conditions of breathing, and gas can be slowly exhaled. Reverse breathing is always adopted in Taijiquan Wujizhuang. The breathing mode is shown as follows: participants exhale and inhale through the nose. The tongue should prop the palate. The lung is fully expanded, thereby the inhaled oxygen can be effectively delivered to the alveoli, thereby increasing the oxygen content of the abdomen. Since a lot of oxygen is inhaled, diaphragmatic muscle is decreased due to extrusion, intra-abdominal pressure is increased to bulge abdomen. Abdomen and lung gas is exhaled in vitro as far as possible. Gas inside the abdomen makes the abdomen contract due to exhalation, and the diaphragmatic muscle is immediately returned in situ. Exhaling and inhaling are uniform and orderly during Wujizhuang standing exercise. Idea import is combined for sinking gas to pubic region. Gas of the whole body is concentrated to pubic region. Inspiratory and expiratory sounds should be intangible, which is beneficial to improve the effect of standing exercise.

Taijiquan Wujizhuang's breathing mode can promote the coordination of internal organs, improve blood circulation, and strengthen the balance between Yin and Yang. It has the functions of 
invigorating blood circulation and enhancing fitness and health. When the body adopts abdominal breathing, the diaphragmatic muscle is lowered due to compression, thereby making the internal organs produce tiny movement, effective circulation of blood and lymph can be promoted under constant movement, thereby improving metabolism, and enhancing the working ability of the internal organs. Oxygen is one of the three largest energies provided for the body. It is important material for metabolism of human body. Brain is an important part of control of the central nervous system of human body. A lot of oxygen supply is required during work. If the oxygen is not supplied timely, or the oxygen is not sufficient, the brain's ability to work will suffer from serious fault immediately. Life will be threatened in serious circumstance. The problem of insufficient oxygen supply can be effectively solved through the abdominal breathing method. After a large amount of oxygen is inhaled, gas can be exchanged sufficiently and effectively in the body, thereby relieving fatigue of our body and stimulating new vitality. Oxygen supply function of heart and lung is also important. Their main function is shown as follows: oxygen is effectively conveyed to every body cell. Body exercise of modern people is seriously declined due to objective factors such as heavy school task, work, etc., thereby leading to acidic and viscous blood, hindering blood circulation, and causing some chronic diseases. Taijiquan Wujizhuang exercise is relatively static. However, effective movement in the body can be regulated through the mode of deep breathing in the process of practice, thereby reducing the acidity and viscosity of blood, achieving the effect of Qi and blood flow, improving the function of cardiovascular system, and preventing heart disease, hypertension, atherosclerosis and other cardiovascular diseases.

\section{Taijiquan Wujizhuang's effect to 'Tiaoxin'}

Tiaoxin refers to the adjustment of mental state so that participants can achieve emotional stability and mind concentration, namely adjustment of mood. It is found that Taijiquan Wujizhuang has very significant effect on Tiaoxin through book and literature review. Taijiquan Wujizhuang gives priority to static state. It is matched with thoughts and breath. Participants can always keep mind concentration and calm down under long-term stationary state, thereby achieving 'comfort' purpose. The exercise can make body comfortable, natural and relaxed, thereby relaxing the nervous system. Meanwhile, it also can relatively relax and adjust all muscles and joints of the body, thereby making people happy.

Tiaoxin is the most important item in Taijiquan standing exercise. Human psychological emotion determines the quality of life, physical health and work efficiency. Relaxed and happy mood can make people positive. People can work and learn hard. Depressing feeling not only can bring resistance to work and study, but also can cause spreading of body disease. In current fast-paced social environment, more and more pressure is brought to people, people should learn to adjust own emotion in high-pressure life, thereby alleviating pressure for themselves. They can study and work better. Tiaoxin function of Taijiquan Wujizhuang is mainly shown as follows: body is relaxed, tension of the nervous system can be adjusted under the precondition of keeping spirit concentrated, thereby calming the heart rate in a quiet state. The exercise can improve the blood supply, and enhance the working ability of nervous system, heart and other organs through the adjustment of mind. It can arouse the enthusiasm of thinking, relieve pressure and body fatigue, etc., thereby people can learn and work actively under full spirit state, and improve the efficiency of study and work.

\section{'Three-in-one' in Taijiquan Wujizhuang}

Three adjustments refer to 'Tiaoshen', 'Pranayama' and 'Tiaoxin'. 'Three-in-one' exercise is one of important adjustment methods to regulate the balance of body Yin and Yang, inner organs, blood circulation and other functions. As the old saying goes: 'Qi is not smooth if the shape is not regular, idea is not peaceful if the Qi is not smooth, and spirit is disorder if the idea is not peaceful'. It is obvious that 'body, spirit and heart' adjustments are complementary mutually and interacted during practice, thereby reaching the effect of 'three-in-one'. 'Three adjustments' are adjusted in Taijiquan 
Wujizhuang. 'Fair comfort' is required in body shape during the practice of Taijiquan Wujizhuang. Breathe is adjusted through Baihui propping, shoulder and elbow dropping, natural looseness and other postures. Breathing should be combined with mind. Participants should feel blood flowing and muscle movement during inhalation and exhalation, thereby leading Qi with will, and people can adjust mind better in quiet state through combining body and breath. 'Tiaoshen, Pranayama and Tiaoxin' in Taijiquan Wujizhuang are interconnected. Overall practice unbalance will be caused if one aspect is not coordinated, thereby all aspects should be mutually cooperated for completing practice, thereby coordinating and developing the body as a whole, and laying a foundation for effective adjustment of 'body, breath and heart'.

'Tiaoshen', 'Pranayama' and 'Tiaoxin' in Taijiquan Wujizhuang have significant fitness and health value to the body. Wherein, 'Tiaoshen' refers to regulation and improvement of body internal and external function, the body quality can be enhanced externally, thereby improving the working ability of muscles and protecting the body. Functions of inner organs can be exercised internally, thereby improving the working ability of all organs. Pranayama refers to the regulation of the body breath, the circulation of blood can be accelerated through the regulation of the breath, thereby promoting metabolism, clearing and activating the channels and collaterals, preventing chronic diseases, etc.. Tiaoxin refers to the regulation of psychological changes in the human body, thereby dredging the emotion, relaxing the mood, and making people energetic and positive. All body parts are fully exercised through long-time cooperation with exercise, thereby enhancing physical quality, promoting muscle flexibility, improving body oxygen intake, increasing the speed of blood circulation, promoting metabolism, regulating channels, improving the working ability of internal organs, relieving fatigue, etc., and it has great effect for the body to prevent diseases and keep healthy body and mind development.

\section{Conclusion}

Taijiquan Wujizhuang practice allows us to 'slow down and calm down' in the fast-paced life, which is also the charm of Taijiquan Wujizhuang itself. Researchers from foreign countries have found that Taijiquan Wujizhuang practice can relieve fatigue, improve sleep quality, lower blood pressure, etc. China is regarded as the source, and researchers discovered the follows through comprehensive research in the aspects of sports physiology, sports psychology,sports anatomy, sports training, etc. for many years: Taijiquan Wujizhuang exercises has fitness and health effect such as fit keeping, smooth breathing, mood regulation, etc. It has the same effect as TCM acupuncture, massage and other therapy aiming at alleviation treatment of some chronic diseases. Taijiquan Wujizhuang, as the therapy means, can be practiced conveniently, expenditure can be saved, and it is undoubtedly a good choice. Taijiquan Wujizhuang is accepted and practiced by more and more people under the environment of vigorously promoting the national fitness and health China, etc. for promoting national physical quality in China. Though certain achievements have development and promotion. It is believed that there is much space for Taijiquan Wujizhuang as an exercise method on the way of promoting human body health.

\section{ACKNOWLEDGMENT}

This paper is supported by the project fund of the Fundamental Research Funds for the Central Universities" (NO. 2017-zy-108)

\section{Reference}

[1] Kang Gewu. Practical Complete Collection of Chinese Martial Arts [M]. Beijing: China Daily Publishing House, 1990.

[2] Wu Tunan. Overview of Chinese Martial Arts [M]. Beijing: China Bookstore, 1984.

[3] Editorial Board of Chinese Martial Arts Dictionary. Chinese Martial Arts Dictionary [M]. 
People's Sports Publishing House, 1990.

[4] Zheng Zhenyou. On Technical Value of Traditional Standing Exercise [J]. Zhongzhou Sports, Shaolin and Taiji, 2009, (2) : 15-17.

[5] Chen Zhihui. Exploration of Health-related Behaviors in 'Huangdi Neijing' [J]. Social Psychological Science, 2006,21 (5) : 75-77.

[6] Feng Jinyong, Chao Yuechun. Research on special role and value of Taiji standing exercise in modern martial arts [J]. Chinese Martial Arts (research), 2012,1(7):39-42.

[7] Duan Yanjin. On value and effect of traditional martial art standing exercise [D]. Henan University, 2008.

[8] Yi Ming. Tiaoshen Definition [EB/OL].http://www.baike.com/wiki/ Tiaoshen .

[9] Tong Jixi. Biomechanical analysis of influence of Taijiquan standing action on spine physiological curvature [D]. Beijing Sports University, 1998.

[10] Wang Xiangzhai, Collected Works of Boxing Master Wang Xiangzhai [M]. Beijing: China Radio and Television Press, 2012.

[11] Yi Ming. Pranayama Definition [EB/OL].http://www.baike.com/wiki/ Pranayama . 